\section{A REPORT OF BACTERIAL VACCINE THERAPY IN A SERIES OF PROSTATIC CASES *}

\author{
H. C. BUMPUS, M.D. \\ Fellow of the Mayo Foundation \\ ROCHESTER, MINN.
}

Following the appearance of the paper by Cabot and Crabtree, ${ }^{1}$ setting forth the favorable results obtained from the administration of colon vaccine in a series of eight prostatic cases, the procedure was adopted from time to time in the Mayo Clinic; but not until May, 1917, was it systematically carried out. At about this time also there appeared an article by von Sholly,
Whittington ${ }^{3}$ reported on 230 typhoid cases with careful controls and, interestingly enough, he also felt confident of favorable results until his careful analysis disclosed again that the controlled patients not receiving the vaccine had done better than those so treated.

In undertaking our study, it seemed advisable to give all the patients the vaccine for five months and then to compare the results with those obtained in the previous five months without the vaccine. Thus the element of coincident time would be sacrificed for that of greater numbers, and the result, we believed, would be more accurate. This also seemed just, since the treatment of these cases is now routine and has not varied appreciably during the present year.

TABIE 1.-FROSTATIC RECORD*

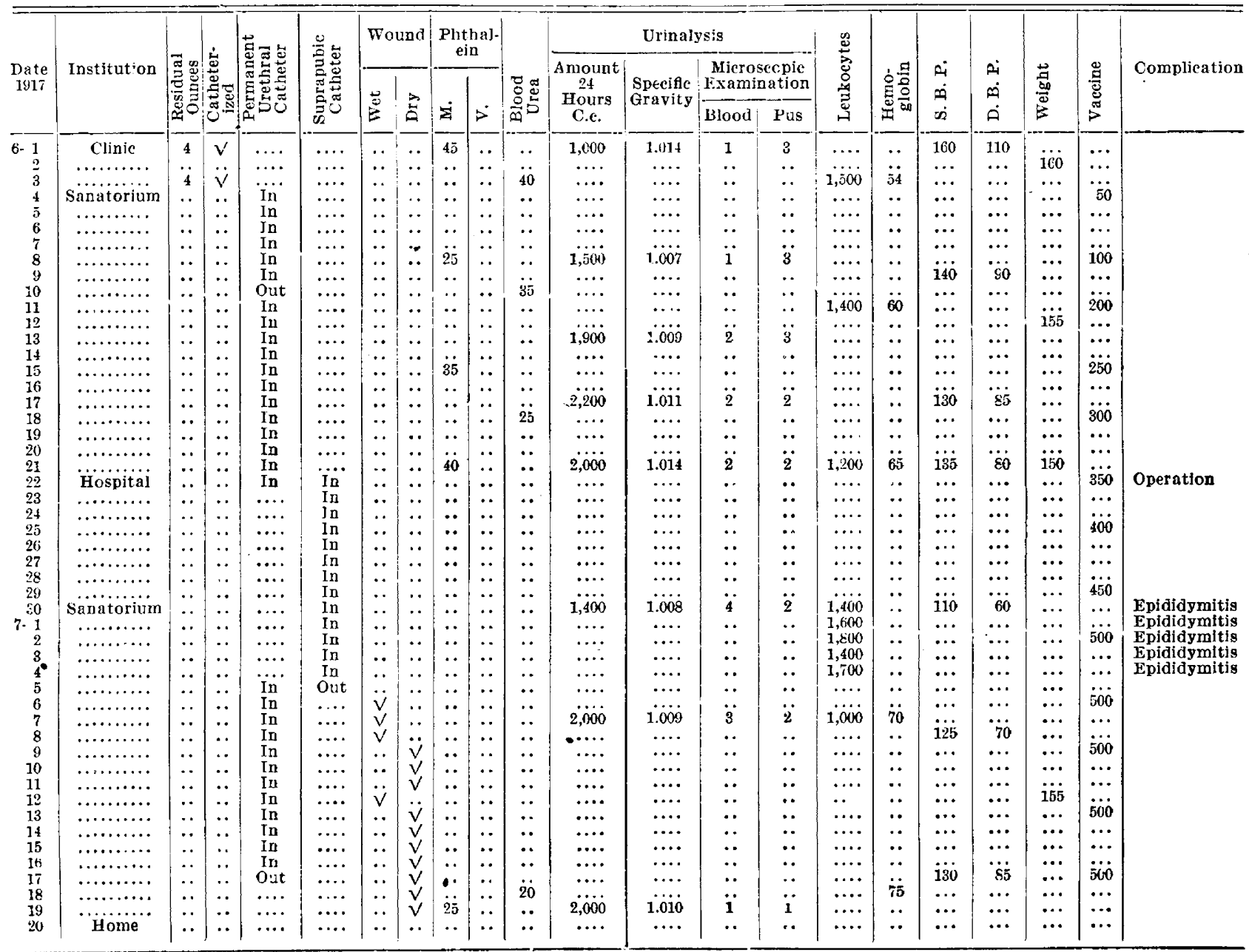

- No. A144495; uge, 66 .

Blum and $\mathrm{Smith}^{2}$ on the therapeutic value of bacterial vaccine in whooping cough, the authors stating that during the administration of the vaccine they were very enthusiastic as to the beneficial results. After their tables had been compiled, however, and carefully compared with the controls, the results showed that the untreated patients had done, on the whole, better than those receiving the vaccine. Similarly,

* From the Mayo Clinic. Non-Tuberculous Renal Infections, Surg., Gynec. and Obst., 1916, 23, 495-537. Crabtree, E. G., and Cabot, Hugh: Colon Bacillus Pyelonephri-
tis: Its Nature and Possible Prevention, TrE Journal A. M. A., Feb. 24, 1917, pp. $589-591$.

2. Von Sholly, Anna I.; Blum, Julius, and Smith, Luella: The Therapeutic Value of Pertussis Vaccine in Whooping Cough, THe Journal A. M. A., May 19, 1917, pp. 1451-1456.
Further to make the two series as synchronous as possible, all cases in which the so-called two-stage operation was done (that is, suprapubic drainage followed by prostatectomy) have been considered separately. Judd ${ }^{4}$ believes that, with the exception of a few selected cases requiring very lengthy drainage to reestablish kidney function, better results are obtainable by means of the permanent urethral catheter than by the suprapubic stab. The former renders a good surgical procedure possible since there is no scar tissue to interfere with ample exposure, thus permitting

3. Whittington, T. H.: A Report on the Use of Stock Vaccine in Infection by the Bacillus Typhosus, Lancet, London, 1916, 1, 759.766. Med. Judd, E. S.: Surgical 
injection of the capsule, excision of the gland, suturing of the capsule, and the establishment of complete hemostasis with ease and precision. The operative field, as in other surgical procedures, is clear and accessible. On the other hand, in the presence of an old suprapubic sinus with its accompanying scar tissue, such procedure is impossible, and the operation that is performed tends to become the finger enucleation of the past with its lamentable lack of precision and total disregard of hemostasis. A permanent urethral catheter preoperatively also has the added advantage of rendering the patient accustomed to its presence, and thereby makes its postoperative use less hazardous.

The routine preoperative and postoperative treatment in relation to the vaccine therapy may be readily understood by Table 1, representing a chart such as is used in our prostatic cases. Thus in parallel columns records are kept of the phenolsulphonephthalein, bloodurea, specific gravity, volume, and microscopic and residual urine, together with the blood pressure, hemoclobin and the amounts of vaccine given from day to day. The vaccine was administered twice a week in doses beginning with 50 million, increasing by multiples of 50 to a maximum of 500 million, and continuing throughout convalescence.

Considerable thought was given to the question of the relative merits of stock and autogenous vaccines. The autogenous vaccines seem to have the greater amount of literature to their credit. They are, of course, always fresher and, on the basis of specificity, would seem to be superior. The stock vaccine, on the other hand, is within reach of the greater number of physicians, and is safer, since it is put out by the large laboratories under federal license. Therefore, following the technic of Cabot and Crabtree, we used mixed colon vaccine prepared from various strains of colon bacillus isolated from different urines and prepared fresh once a week.

The administration was subcutaneous and, with two exceptions, no untoward symptoms resulted. The majority of the patients complained of malaise with slight nausea and a little rise of temperature during the first few inoculations. The reactions rescmbled those usually associated with the administration of typhoid vaccine. Some patients stated that they could feel no effect; the majority regarded it as a necessary evil, and complained most of the localized inflammatory reaction, which lasted one or two days but always wore off. In the two patients mentioned, the reaction was marked enough to require abandonment of the treatment, but never was in any way alarming.

To determine our results, a comparison of the patients treated with vaccine with those not receiving vaccine must be made relative to the incidence of complication manifestly due to infection, namely, pyelonephritis, epididymitis, phlebitis, cystitis and wound infection. The last two conditions it seems best to disregard, as both are almost universally present to a mild degree in such cases, and their relative severity is difficult to use as a matter of careful comparison.

Pyelonephritis is naturally our best criterion. The characteristic symptoms of malaise, accompanied for several days by gradually increasing fever with chills and sweats and a subsequent decline, make it typical; and to one who has been long associated with patients who are having residual urine repeatedly removed, the so-called "reaction" can never be forgotten. Cabot and Crabtree have reported a series of cases in which the colon bacillus was obtained during such reactions, especially during the chills, in pure culture from the blood stream. We attempted to repeat their experiments, and although blood was obtained from six patients, all within an hour of the onset of the chill and from two at its height, in no instance could a growth be obtained, ont cultures always being sterile. Owing to the absence of Cabot and Crabtree in Europe, we were unable to ascertain wherein our technic differed from theirs, and so gave up further attempts to isolate the organism. Therefore, to prevent such reactions or to reduce their severity becomes the chief function of the vaccine, and its final sentence in the court of last appeals must be based largely on its ability to produce evidence that the incidence of pyelonephritis is lessened by its administration. It has long been known and fully realized that a patient having undergone such a reaction, although for the time greatly weakened, is a better surgical risk than he who has not. Therefore, a patient having had a reaction and being subsequently treated with colon vaccine may well be likened to one having recovered from typhoid and subsequently receiving typhoid prophylactic treatment, unless the immunity conferred by an attack of pyelonephritis is only transient, as Cabot and Crabtree suggest.

Phlebitis and epididymitis have also been added, together with the length of preoperative treatment and of convalescence, but the main judgment must rest on the pyelonephritis incidence. It seems only fair to state that at the writing of this paper the results had not been compiled, that at first we were very enthusiastic, that subsequently we became skeptical, and that after the completion of Table 2 , we discontinued the administration of the vaccine.

TABLE 2.-PROSTATECTOMIES FROM JAN. 1, 1917, TO NOV. 1,1917

\begin{tabular}{|c|c|c|c|c|}
\hline \multirow[b]{2}{*}{ - } & \multirow{2}{*}{$\begin{array}{l}\text { For All } \\
\text { Causes }\end{array}$} & \multicolumn{3}{|c|}{$\begin{array}{l}\text { For Adenofbromatous } \\
\text { Hypertrophy }\end{array}$} \\
\hline & & $\begin{array}{l}\text { Reeciving } \\
\text { Vaecine }\end{array}$ & $\begin{array}{c}\text { Not } \\
\text { Receiving } \\
\text { Vaceine }\end{array}$ & $\begin{array}{l}\text { Two-stage } \\
\text { Operation }\end{array}$ \\
\hline Number............... & 105 & 49 & 24 & 12 \\
\hline Average age................ & (ii) & 6 & $6 i$ & 62 \\
\hline Days prior to operation.... & 31 & 38 & 23 & 279 \\
\hline Days of convalescence...... & 34 & 32 & 37 & 28 \\
\hline Total days treated......... & 6 & 70 & 60 & 307 \\
\hline Pyelonephritis............... & $31 \%$ & $3 \% \%$ & $37 \%$ & $\ldots$ \\
\hline Epiāidymitis............... & $20 \%$ & $35 \%$ & $\overline{2} \%$ & ... \\
\hline Phlebitis................... & $4 \%$ & $4 \%$ & $4 \%$ & ... \\
\hline Number of deaths in the & & & & \\
\hline hospital.................. & 2 & $\mathbf{1}$ & 0 & 1 \\
\hline
\end{tabular}

'Thus it appears that in the series in which vaccines were given, therc was 5 per cent. less of cases of pyelonewhritis than the controls, and 1 per cent. more than all cases. However, it must not be concluded that the vaccine has produced this decrease; for if the action of the vaccine were specific, a greater reduction than 5 per cent. should have occurred, and the incidence, as compared with all patients operated on, should have been less - not grcater. This conclusion is further supported by the 10 per cent. increase in the cases of epididymitis among patients that were treated compared with the controls.

A survey of the length of time the treatment was given is of interest in demonstrating that, although the convalescence of the patients not receiving vaccine is longer by five days, their entire time under treatment is shorter by ten days, showing that the greater the length of preoperative preparation the shorter the con- 
valescence. This fact is further emphasized in the two-stage series, when a preparation of several hundred days is given to patients who are the poorest of surgical risks, and leads to a convalescence much shorter. than the average. In the two-stage series no attempt was made to chart the incidence of infection, because much of the patients' preoperative time was spent at their homes, where accurate observation was, of course, impossible.

\section{CONCLUSIONS}

1. Immunity to pyelonephritis by means of mixed colon bacillus vaccine cannot be produced.

2 . The administration of mixed colon vaccine does not markedly reduce the incidence of genito-urinary infection, if it affects it at all.

3. Preoperative attacks of pyelonephritis are the natural means of producing an immunity to renal infection, and their occurrence makes operative risks . less.

4. The length of convalescence is usually in inverse proportion to the length of preoperative treatment.

\section{THE OCCURRENCE OF A POSITIVE INTRACUTANEOUS REACTION IN MENINGOCOCCUS CARRIERS *}

\author{
FREDERICK P. GAY, M.D. \\ BERKELEY, CALIF. \\ AND \\ A. J. MINAKER, M.D. \\ Assistant Surgeon, U. S. Naval Reserve Force \\ SAN FRANCISCO
}

The importance of any facts bearing on meningococcus carriers makes advisable a brief report on our present limited series of observations with an intradermal test in meningococcus carriers, in order that they may be extended by others who are dealing with this problem.

It would probably be impossible to make any conclusive statement at present as to the degree of systemic reaction of healthy individuals who harbor the meningococcus in their throats to this micro-organism. On the degree of this reaction would depend, of course, their resistance to further invasion of the coccus; and concerning this resistance we have conflicting testimony. Temporary carriers may, subsequent to the bacteriologic detection of the meningococcus in their throat, fall ill of meningitis; on the other hand, the majority of detected carriers certainly are themselves unaffected in any serious manner by the parasite they harbor. There is, indeed, certain evidence from the occurrence of meningismus or aborted attacks of meningitis in carriers, as noted by Flack, ${ }^{1}$ that an acquired immunity of some efficiency has been engendered in at least some of the carriers which protects them partially from specific meningitis.

It has been generally admitted and specifically proved by one of us (A. J. M.) that the usual tests for antibodies (agglutinins, fixation antibodies) yield no results of significance in positive meningococcus carriers. It seemed of interest, however, particularly

* From the Department of Pathology and Bacteriology of the University of California and the U. S. Naval Training Station, Yuba Buena Island, San Francisco.

1. Flack, M.: Report on Cerebrospinal Fever in the London District, December, 1915-July, 1916, Special Report of the Indicated Research Committee on Bacteriological Studies in the Pathology, Prevention and 1916, London, T. Fisher Unwin, 1917. in view of the results of the typhoidin test in cases of recovery from typhoid fever, to test the localized intracutaneous susceptibility to preparations of the meningococcus in positive carriers, as compared with those who, on similar bacteriologic examinations from the pharynx, presented no meningococci in detectable numbers. For several months the naval recruits at this station have been systematically examined for the presence of the meningococcus, and positive carriers have been isolated until cultures from them became negative, following appropriate treatment. In connection with this particular investigation, it should be specifically stated that it cannot be assumed that this separation between positive and negative carriers on the basis of a single bacteriologic examination is perfect. It can, indeed, be assumed from work on this subject that among those classed as positive meningococcus carriers were included some individuals who harbored only gram-negative and nonpathogenic chromogens instead of meningococci, and on the other hand that those who were dismissed as "negative" must, in some cases, have harbored meningococci.

The preparation of meningococcus ("meningococcin") used in these experiments was made as follows: Pure cultures of five of the six strains chosen as representative of the meningococcus group by Amoss (Rockefeller strains numbered 1, 10, 30, 44 and 60 , including two normal meningococci ; two irregulars, and one normal parameningococcus) were grown on 1 per cent. starch agar without peptone for fortyeight hours in ordinary slant tubes. These separate cultures were suspended in sterile saline solution, to each tube 3 c.c. The suspended cultures were mixed, and three volumes of absolute alcohol added. The organisms were flocculated at once and were rapidly centrifugalized, the supernatant fluid removed, and the bacterial deposition thoroughly shaken in the original volume of fresh absolute alcohol. Recentrifugalization, removal of alcohol, and resuspension in ether (distilled over sodium) were performed. After recentrifugalization and decantation, the sediment was dried two days in partial vacuum over sulphuric acid. The hard dried sediment was ground to impalpable powder in an agate mortar and dried again for twentyfour hours as before.

This preparation was then suspended in physiologic sodium chlorid solution containing 0.5 per cent. phenol (carbolic acid). The dose of this suspension, which tends gradually to sediment, employed in the intradermal tests is a volume of 0.05 c.c. containing $1 / 150 \mathrm{mg}$. $(0.000006+\mathrm{gm}$.$) of the dried meningococcus pow-$ der. Great care is taken to inject this dose with a fine (27 gage) hypodermic needle so as to leave a persistent bleb under the superficial layers of the skin.

Readings of the reaction were made at the six hour, twenty-eight hour and forty-eight hour periods. A positive reaction consists in a well demarcated areola of from 3 to $7 \mathrm{~mm}$. which is distinctly indurated. The most distinctive results are apparently present at the twenty-four-twenty-eight hour period, and the reaction has usually disappeared in forty-eight hours. We have found this reaction positive between two and three times as frequently in those classified by bacteriologic examinations as positive when compared with those classified negative, thus:

In thirty-one positive carriers, the intradermal test was positive in twenty, or 64.5 per cent.

In thirty-eight negative carriers, the intradermal test was positive in ten, or 26.4 per cent. 